\title{
Die Wertigkeit der bildgebenden Verfahren an Schulter und Oberarm
}

\author{
Honke G. Hermichen, Rainer Köster, Lothar Mansfeld
}

\section{Zusammenfassung}

Die modernen Entwicklungen der bildgebenden Verfahren an Schulter und Oberarm haben die differenzierte Therapie von Verletzungen und degenerativen Veränderungen in diesem Bereich erst möglich gemacht. Röntgenaufnahmen, Sonographie, Computertomographie sowie Kernspintomographie haben ihren festen Platz in der Diagnostik gefunden. Durch diese Untersuchungstechniken sind wir heute in der Lage, früher nicht darstellbare Strukturen sichtbar zu machen. Die verschiedenen Methoden haben bestimmte teilweise sich überlappende Indikationsbereiche und bedürfen daher einer individuell abgestimmten Anwendung.

\section{Einleitung}

In den letzen Jahren hat die bildgebende Diagnostik an der oberen Extremität einen großen Aufschwung erfahren. Davon hat gerade die differenzierte Schulterchirurgie erheblich profitiert. Bislang schwierig zu identifizierende Strukturen sind radiologisch darstellbar geworden. Dies erleichtert die Krankheitserkennung wie auch die Auswahl der unterschiedlichen Therapieoptionen.

Umso wichtiger ist es aber jetzt, die verschiedenen Techniken auch verletzungsund krankheitsädaquat einzusetzen.

Nur durch Kenntnis der Stärken und Schwächen des jeweiligen Verfahrens

OP-JOURNAL 2002; 18: 190-193

(c) Georg Thieme Verlag Stuttgart · New York kann im Einzelfall das sinnvolle und rationelle diagnostische Procedere festgelegt werden.

\section{Das Röntgenbild}

Röntgenaufnahmen der betroffenen Körperregion in 2 Projektionsebenen mit routinemäßiger Abbildung des angrenzenden Gelenkes (hier: Schultergelenk) sind nach wie vor der „Goldstandard“ in der Primärdiagnostik (Abb. 1, 2).

Diese Bilder sind bei Verdacht auf eine knöcherne Verletzung, eine Luxation wie auch bei Verschleißerkrankungen oder Tumorverdacht unverzichtbar (Abb. 3,4).

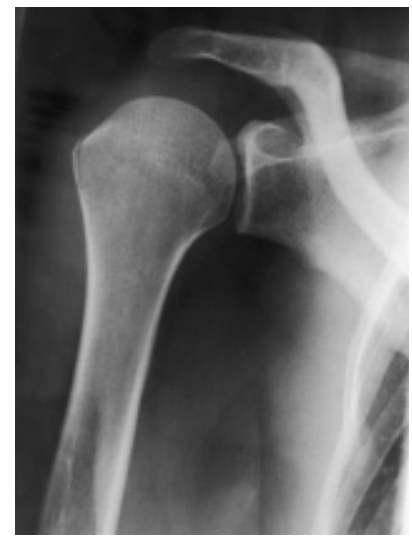

Abb. 1 Rö-Bild li Schulter, Infraktion Tub. majus.

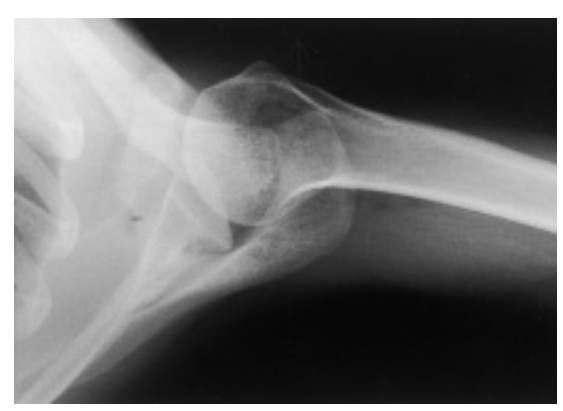

Abb. 2 Axialaufnahme li Schulter.
In der wissenschaftlichen Fachliteratur existiert eine Vielzahl von Empfehlungen über die aussagekräftigsten Bildebenen, Aufnahmetechniken, Spezial- und Funktionsaufnahmen und vieles mehr. Wichtig ist als Basis die Abbildung in 2 Projektionsebenen, um den „Körper“ Knochen

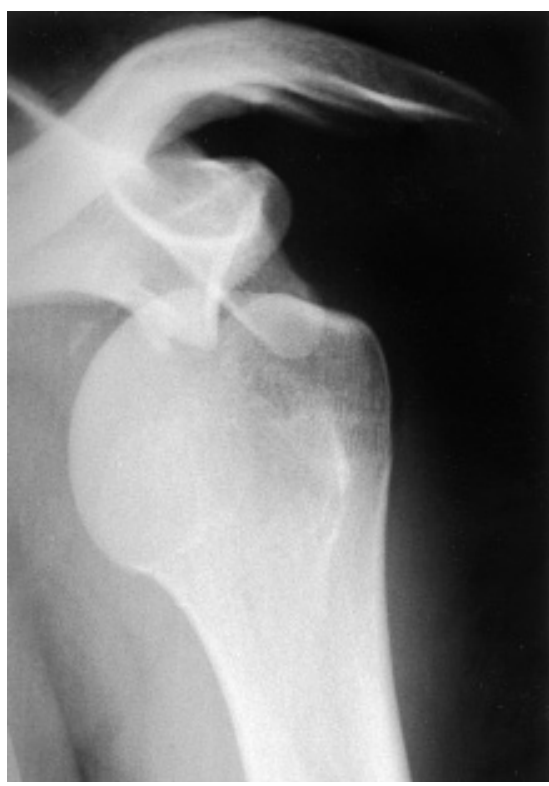

Abb. 3 Schulterluxation (beachte die leere Pfanne!).

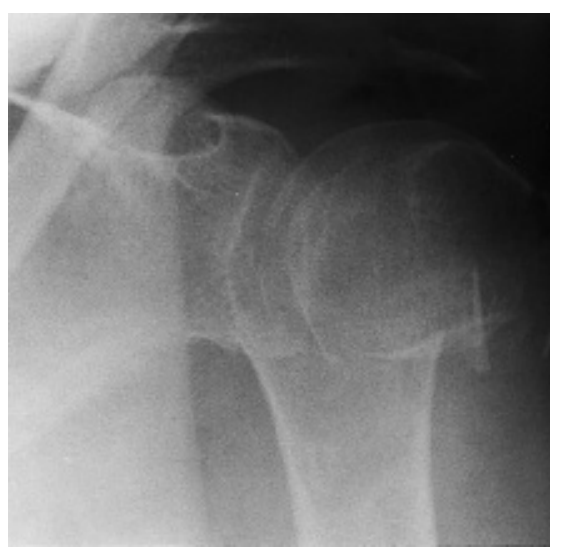

Abb. 4 Oberarmkopffraktur bei schwerer Osteoporose. 


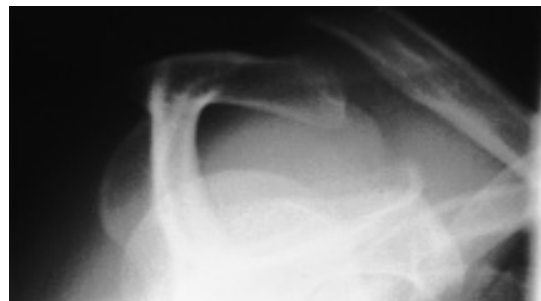

Abb. 5 Spezialaufnahme der lateralen Klavikula: Luxation im AC-Gelenk („Rockwood IVVerletzung“).

und Gelenk in der Fläche abzubilden. Nur so werden Fehlinterpretationen vermieden. Welche spezielle Technik im Einzelfall gewählt wird, ist abhängig von vielen Faktoren, deren Aufzählung jetzt aus Platzgründen nicht möglich ist (Abb.5).

Wichtig ist jedoch die routinemäßige Anwendung von etablierten Standardaufnahmen. Nur so kann auch die notwendige Erfahrung des Untersuchers gewonnen werden. Eine vertrauensvolle und informative Zusammenarbeit zwischen dem Kliniker und dem Radiologen trägt sicher dazu bei, Qualitätsstandards zu entwickeln und auch beizubehalten.

Zu den erwähnten Spezialaufnahmen zählen beispielsweise die häufig angefertigten „Aufnahmen mit Belastung“ zur Erkennung von Instabilitäten im Schultereckgelenk („AC-Gelenk-Sprengung“) (Abb.6).

Frakturen im Bereich des Oberarmschaftes sind eine Domäne der Röntgendiagnostik (Abb. 7). Dieses gilt gleichermaßen für die Verlaufsbeurteilung nach einer Osteosynthese oder auch nach konservativer Behandlung (Implantatposition, Stabilität, Kallusbildung knöcherne Ausheilung) (Abb.8).

Das Röntgenbild stellt nicht nur den Knochen dar - viele Hinweise auf den Zustand der umgebenden Weichteile sind vorhanden und auch in zahlreichen Bildern sichtbar (z. B. Lufteinschlüsse bei offenen Verletzungen, Hochstand des Oberarmkopfes bei chronischer Rotatorenmanschettenruptur und Impingement (Engpass)-Syndrom).

Dennoch sind meist nur indirekte Rückschlüsse auf den Zustand der Weichteile möglich. Hier bietet sich als weiterführende Diagnostik die Sonographie des Schultergelenkes an.
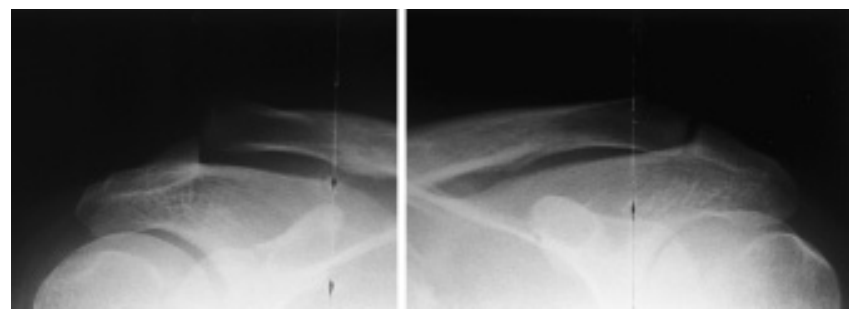

Abb. 6 AC-Sprengung re, Aufnahme mit Belastung im Seitenvergleich re-li.

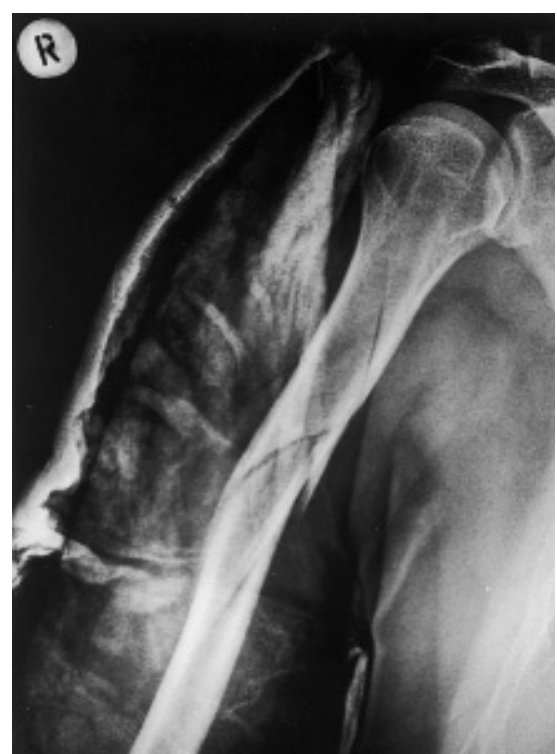

Abb. 7 proximale Humerusschaftfraktur, Gipsüberlagerung.

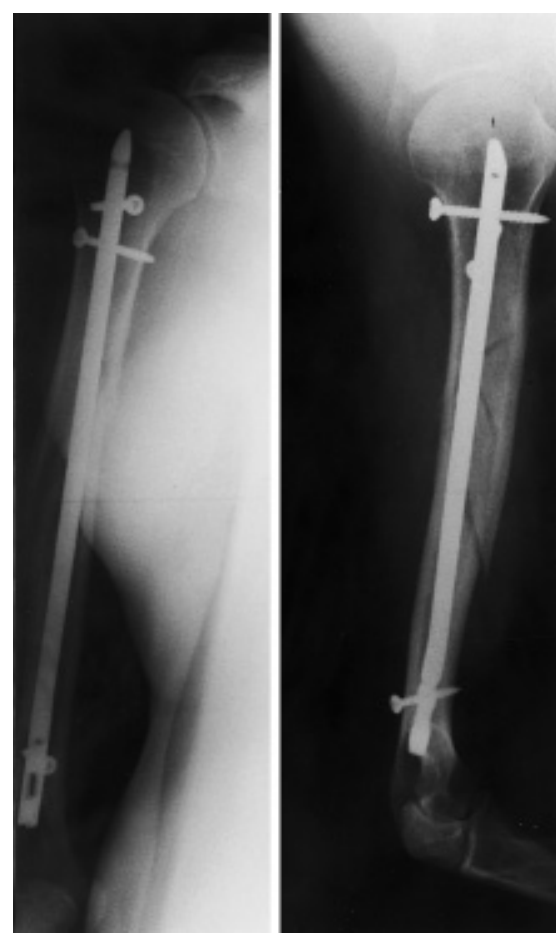

Abb. 8 Humerusmarknagel (UHN) mit Abbildung von Schulter- und Ellbogengelenk.

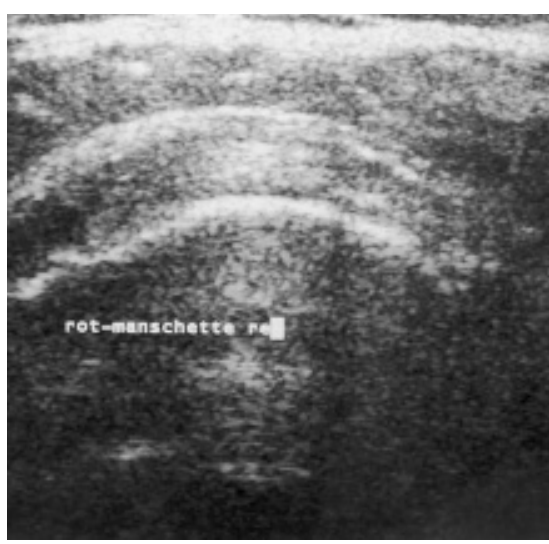

Abb.9 Sonographie der (intakten) Rotatorenmanschette.

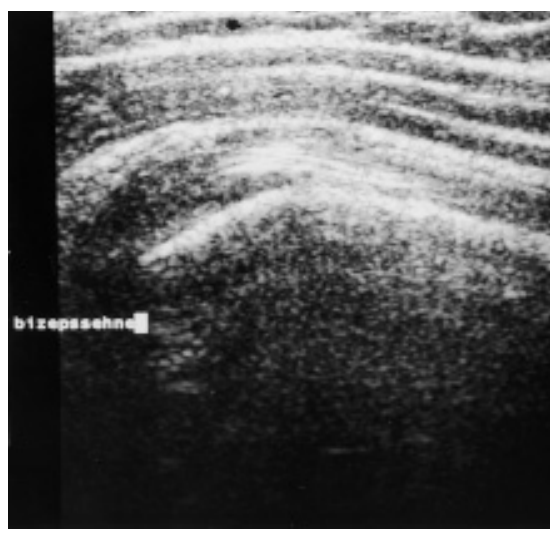

Abb.10 sonographische Darstellung der langen Bizepssehne.

\section{Ultraschalluntersuchung - Sonographie}

Bedingt durch die Entwicklung hochempfindlicher Schallköpfe ist die Sonographie an der Schulter zu einem wichtigen Diagnostikum gerade bei der Beurteilung der Weichteile geworden. Der große Vorteil liegt in der nicht vorhandenen Strahlenbelastung, der Nichtinvasivität sowie der eigentlich unbeschränkt möglichen Wiederholung dieser funktionellen Untersuchung. Bei entsprechender Erfahrung lassen sich mit der Sonographie Läsionen der Rotatorenmanschette, der Bizepssehne und anderer Weichteilstrukturen zuverlässig nachweisen (Abb. 9,10). 
Auch Schleimbeutelverdickungen oder Verkalkungen im Sehnenbereich sind darstellbar.

Der Begriff „funktionelle Untersuchung“ besagt, dass die Sonographie im Bewegungsablauf des Schultergelenkes (aktiv oder passiv) ihre größte Aussagekraft entfaltet.

Die Untersuchung findet meist im Sitzen unter ruhigen äußeren Bedingungen statt. Fast immer ist der Seitenvergleich z.B. zur Bestimmung der Relation zwischen Humeruskopf und Glenoid sowie Akromion hilfreich und erforderlich.

Die technische Entwicklung geht auch bei der Schultersonographie rasant voran, so dass in der Zukunft dieses Verfahren noch eher an Bedeutung gewinnen dürfte.

Bei Verletzungen im Schaftbereich des Oberarms kann die Ultraschalluntersuchung z.B. bei der Größenbeurteilung eines postoperativ aufgetretenen Hämatoms gute Dienste leisten. Auch die Darstellung der Knochenbruchheilung ist heutzutage in geübter Hand durch die Sonographie möglich. Diese Techniken sind aber zur Zeit noch nicht so aussagekräftig und valide wie das Röntgenbild. Sie gewinnen aber zunehmende Bedeutung bei der Beurteilung kindlicher Frakturverläufe und vermindern dadurch die Strahlenbelastung der Patienten.

Wichtig ist bei der Sonographie eine präzise und reproduzierbare Befunddokumentation. Bilder allein reichen hier im Regelfall nicht aus. Sie bedürfen der Ergänzung durch einen entsprechenden Bericht. Der standardisierte Untersuchungsgang hilft, Fehlinterpretationen zu vermeiden.

\section{Computertomographie (CT)}

Durch das CT mit modernen Schnitttechniken (z.B. „Spiral-CT“) können v.a. im Standardröntgenbild auffällige aber nicht genau zuzuordnende Befunde weiter zuverlässig abgeklärt werden. Wichtig ist auch hier der Hinweis auf die Funktionalität dieser Untersuchung. Durch Kontrastmittelgabe oder Luftinsufflation in das Schultergelenk (sog. „Arthro-CT“) erweitert sich die Beurteilbarkeit auch auf die das Gelenk umgebenden Weichteilstrukturen (Abb.11).

Der Hauptvorteil des CT liegt aber zweifelsfrei in der exakten Feinbeurteilung der Verletzungen oder sonstigen pathologischen Prozesse am Knochen.
Hier sind insbesondere die Befunde an der Gelenkpfanne („Glenoid“) von Bedeutung (Abb.12). Auch die Beurteilung mancher Trümmerfrakturen am Oberarmkopf ggf. mit einer CT-Rekonstruktion können die Therapieentscheidung (z.B. kopferhaltender Eingriff mit Osteosynthese oder Prothesenimplantation) erleichtern. Mit Hilfe des CT können (im Seitenvergleich) die korrekten Winkel von Humerusschaft zu Humeruskopf ermittelt werden. Dieser Aspekt hat Bedeutung bei der Frakturversorgung wie auch bei der Planung von korrigierenden Osteotomien. Bei degenerativen Veränderungen kommt das CT nicht mehr so häufig zur Anwendung. Hier bietet die MRTUntersuchung erhebliche Vorteile.

Im Schaftbereich des Humerus spielt das CT in der Regel keine wesentliche Rolle. Hier reichen fast immer die Röntgenaufnahmen zur genauen Analyse und Therapieplanung aus.

\section{Kernspintomographie (MRT, NMR)}

Das MRT hat v. a. in der differenzierten Schulterdiagnostik dazu beigetragen, wesentliche Strukturen sichtbar zu machen, die sich bislang der Darstellung entzogen (Abb.13).

Hier sind insbesondere die einzelnen Bänder und Sehnen im Bereich des Schultergelenkes zu nennen. Partial- oder Totalrupturen der Rotatorenmanschette, Affektionen der langen Bizepssehne, frische oder ältere Verletzungen des Labrum glenoidale lassen sich mit dieser Methode darstellen. Erst durch diese Bildgebung ist dann auch die entsprechende exakte und bildgestützte Diagnose zu stellen (Abb.14). Okkulte Frakturen („bone bruise“) lassen sich mit dem MRT besser als mit dem konventionellen Röntgenbild nachweisen. Bei degenerativen Veränderungen wird ein MRT dann mit Vorteil einzusetzen sein, wenn durch die Sonographie bereits entsprechende Befunde erhoben wurden. Das MRT dient dann der „Feindiagnostik“ und unterstützt die Therapieplanung. Auch bei der MRT-Untersuchung kann durch Kontrastmittelgabe eine noch höhere Aussagekraft erzielt werden.

Das MRT stellt zweifellos das teuerste und auch derzeit noch zeitaufwändigste diagnostische Verfahren dar - die fehlende Strahlenbelastung (anders als beim CT) ist allerdings gerade bei häufigen Untersuchungen von Vorteil. Die Darstellung

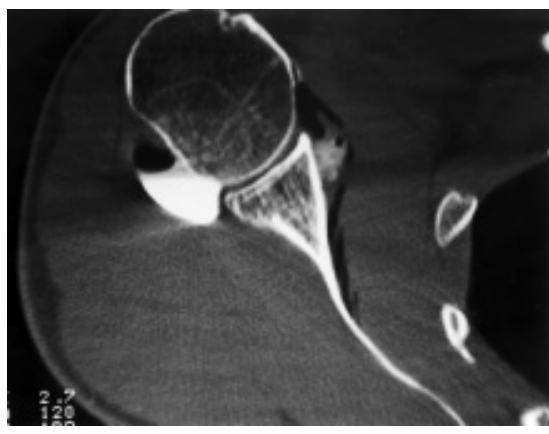

Abb.11 Arthro-CT der Schulter.

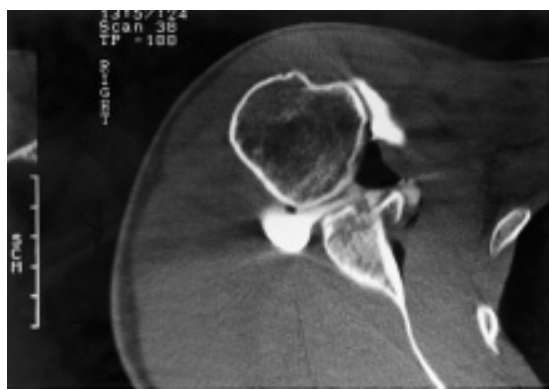

Abb.12 CT-Darstellung Fraktur des Glenoids („Bankart-Läsion“).

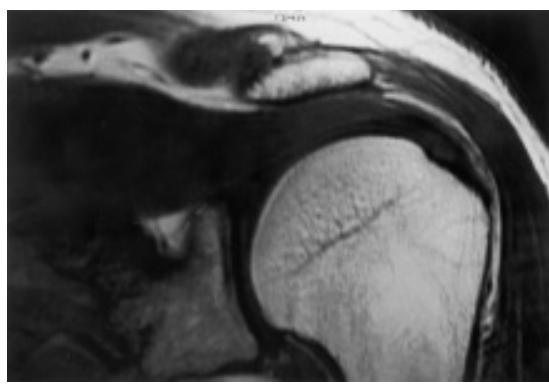

Abb.13 MRT der Schulter, intakte Rotatorenmanschette.

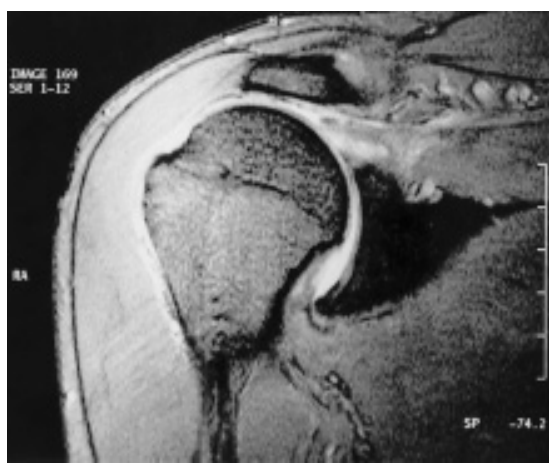

Abb.14 MRT mit schwerem Defekt der Rotatorenmanschette, Hochstand des Oberarmkopfes (vergleiche Abb.13). 
der knöchernen Strukturen gelingt im Regelfall jedoch mit dem CT besser (s. o.). Im Schaftbereich des Oberarmes gibt es - abgesehen bei Tumorverdacht oder anderen seltenen Fragestellungen - derzeit kaum eine Indikation, ein MRT durchzuführen.

\section{Rationelles Vorgehen in der Klinik}

In Klinik und Praxis stellt sich häufig die Frage, wann welche Untersuchung beim Patienten durchzuführen ist. Es ist nicht möglich, hier einen allgemeingültigen $\mathrm{Al}$ gorithmus aufzustellen, da eben die verschiedenen Techniken „Stärken und Schwächen“ aufweisen. Hinzu kommt, dass nicht überall auch alle apparativen Voraussetzungen gegeben sind.

Schließlich sollte auch der wirtschaftliche Aspekt Erwähnung finden: nicht bei jeder frischen Schulterverletzung ist ein MRT oder CT als Primärdiagnostik angezeigt.
Die große Variationsbreite der pathologischen Prozesse am Schultergürtel und am Oberarm bedingt nach der eingehenden klinischen Untersuchung eine individuell abgestimmte bildgebende Diagnostik.

Als generelle „Faustregel“ gilt: initial werden in den meisten Fällen zunächst die Standard-Röntgenbilder angefertigt, ggf. ergänzt durch spezielle Zielaufnahmen. Als 2.Stufe folgt im Regelfall die Sonographie. Die aufwändigeren apparativen Untersuchungen (CT, MRT) werden danach auf den Einzelfall abgestimmt indiziert. Durch ein derartiges stufenweises Vorgehen lassen sich einmal überflüssige, aufwändige und kostspielige Untersuchungen vermeiden, andererseits kann die Diagnostik die notwendige Genauigkeit gewährleisten.

\section{Literatur}

1 Habermeyer P. Schulterchirurgie. Urban \& Fischer, München Jena 2002

2 Mäurer J. Effiziente Schulterbildgebung. Ein Update für den klinischen Alltag. Thieme, Stuttgart 2002

3 Vahlensieck M, Reiser M. MRT des Bewegungsapparats. Thieme, Stuttgart 1997

\section{Dr. med. Honke G. Hermichen}

Chefarzt

Chirurg. Klinik II (Klinik für Unfall-

u. Wiederherstellungschirurgie)

Prof. med. Dr. Rainer Köster

Chefarzt

Dr. med. Lothar Mansfeld

Oberarzt

Institut für klinische Radiologie

Städt. Kliniken Neuss Lukaskrankenhaus GmbH

Preußenstr. 84

D-41464 Neuss 